\title{
Combination Treatment With Sorafenib and Everolimus Regresses a Doxorubicin-resistant Osteosarcoma in a PDOX Mouse Model
}

\author{
TAKASHI HIGUCHI ${ }^{1,2,3}$, NORIHIKO SUGISAWA ${ }^{1,2}$, KENTARO MIYAKE $^{1,2}$, \\ HIROMICHI OSHIRO ${ }^{1,2}$, NORIO YAMAMOTO ${ }^{3}$, KATSUHIRO HAYASHI $^{3}$, HIROAKI KIMURA $^{3}$, \\ SHINJI MIWA ${ }^{3}$, KENTARO IGARASHI ${ }^{3}$, ZOEY KLINE $^{4}$, PAIGE BELT $^{4}$, SANT P. CHAWLA $^{5}$, \\ MICHAEL BOUVET ${ }^{2}$, SHREE RAM SINGH ${ }^{4}$, HIROYUKI TSUCHIYA ${ }^{3}$ and ROBERT M. HOFFMAN ${ }^{1,2}$ \\ ${ }^{1}$ AntiCancer, Inc., San Diego, CA, U.S.A.; \\ ${ }^{2}$ Department of Surgery, University of California, San Diego, CA, U.S.A.; \\ ${ }^{3}$ Department of Orthopedic Surgery, Kanazawa University, Kanazawa, Japan; \\ ${ }^{4}$ Basic Research Laboratory, National Cancer Institute, Frederick, MD, U.S.A.; \\ ${ }^{5}$ Sarcoma Oncology Center, Santa Monica, CA, U.S.A.
}

\begin{abstract}
Background/Aim: Osteosarcoma is a rare but recalcitrant type of bone cancer. To discover an effective therapy for osteosarcoma, we used a patient-derived orthotopic xenograft (PDOX) mouse model. A PDOX mouse model has been established for all major cancer types. Strong synergistic efficacy of sorafenib (SFN) and everolimus (EVL) has been demonstrated in several cancers. In the present study, we examined the efficacy of a SFN and EVL combination on a doxorubicin (DOX)-resistant osteosarcoma PDOX. Materials and Methods: The osteosarcoma PDOX models were randomly divided into five treatment groups, each containing six mice: Control; DOX; SFN; EVL; and a combination of SFN and EVL. Mice were treated for 14 days. To observe the efficacy of these treatments, tumor size and body weight were measured, and histological sections were analyzed. Results: Tumor growth regression was observed only in the mice treated with the combination of SFN-EVL. Histological analysis revealed necrosis with degenerative changes in tumors treated with a combination of SFN-EVL. Conclusion: A SFN-EVL combination could be a novel effective treatment option for osteosarcoma.
\end{abstract}

Correspondence to: Robert M. Hoffman, Ph.D., AntiCancer, Inc., 7917 Ostrow Street, San Diego, CA 92111, U.S.A. Tel: +1 8586542555, Fax: +18582684175, e-mail: all@anticancer.com; Shree Ram Singh, Ph.D., Basic Research Laboratory, National Cancer Institute, Frederick, MD, 21702, U.S.A. Tel: +1 3018467331, e-mail: singhshr@mail.nih.gov; Hiroyuki Tsuchiya, M.D., Ph.D., Department of Orthopaedic Surgery, Kanazawa University, 13-1, Takara-machi, Kanazawa 920-8641, Japan. Tel: +81 0762652374, email: tsuchi@med.kanazawa-u.ac.jp

Key Words: Osteosarcoma, sorafenib, everolimus, drug resistance, PDOX, combination therapy, tumor regression.
Osteosarcoma is a rare type of malignancy. More than 800 new cases of osteosarcoma are diagnosed in the United States each year (American Cancer Society, Cancer Facts \& Figures 2019. Atlanta, Ga. American Cancer Society; 2019). Advances in multimodal therapy have improved the survival rates by more than $65 \%$ for patients with localized osteosarcoma (1). However, recurrent and progressive osteosarcoma is a recalcitrant disease.

Sorafenib (SFN), an inhibitor of various tyrosine protein kinases $(2,3)$, reduces tumor microvessel density and shows anti-proliferative activity in various solid tumors (4-7).

Everolimus (EVL) is the mammalian target of rapamycin (mTORC1)-specific inhibitor (8). The phosphati-dylinositol3 kinase (PI3K)-Akt-mTOR signaling pathway that controls cell proliferation, autophagy, resistance to apoptosis, and tumor development in various human cancers including osteosarcoma $(8,9)$.

EVL has been shown to increase the activity of SFN by targeting different intracellular targets and can reverse resistance to SFN. Synergistic interactions between SFN and EVL have been shown in studies of several cancers such as renal, hepatic, and pancreatic cancers (4-7, 9-11).

In the present study, we tested the efficacy of the SFN-EVL combination on doxorubicin (DOX)-resistant patient-derived orthotopic xenograft (PDOX) mouse model of osteosarcoma.

\section{Materials and Methods}

Animals. In the present study, we used athymic nu/nu nude mice (AntiCancer, Inc., San Diego, CA, USA). The mouse investigations were conducted in compliance with an AntiCancer, Inc. Institutional Animal Care and Use Committee (IACUC) protocol exclusively approved for this study $(12,13)$. All the animal studies were conducted according to the principles and procedures described in 
the National Institutes of Health (NIH) Guide for the Care and Use of Laboratory Animals under Assurance Number A3873-1 $(12,13)$. During surgery, suffering of the animals were minimized using anesthesia and analgesics $(12,13)$.

Patient-derived tumor. A fresh biopsy sample of a 14-year-old boy with pelvic osteosarcoma was used $(13,14)$. No chemotherapy or radiotherapy was given to the patient before biopsy. The patient provided written informed consent as part of a UCLA Institutional Review Board approved protocol (IRB\#10-001857) (13). The patient tumor sample was cut into 5-mm fragments and individual pieces were subcutaneously implanted into nude mice (13).

Establishment of an osteosarcoma PDOX model. Anesthesia to the mice was performed as previously described (14). Harvesting of subcutaneous-implanted tumors, their fragmentation, and implantation into the mouse's distal femur, and wound closure to establish an osteosarcoma-PDOX model were performed as previously described (13-15) (Figure 1A).

Treatment scheme. The osteosarcoma PDOX mouse models were randomly divided into 5 groups (Figure 1B). Each group contained six mice. Mice were treated with the following drugs for 2 weeks (Figure 2B): Group 1 (G1), untreated control; Group 2 (G2), DOX ( $3 \mathrm{mg} / \mathrm{kg}$, intraperitoneal [i.p.] injection, once a week) alone; Group 3 (G3), SFN (30 mg/kg, oral, daily) alone; Group 4 (G4), EVL (5 $\mathrm{mg} / \mathrm{kg}$, oral, daily); Group 5 (G5), SFN (30 mg/kg, oral, daily) + EVL (5 mg/kg, oral, daily). Treatment started once tumor size became $100-200 \mathrm{~mm}^{3}(14,15)$. Tumor length, and width, and body weight of the mouse were measured twice/ week $(14,15)$. Tumor volume was calculated based on our previous publications $(14,15)$. Data are expressed as mean \pm standard error of the mean (SEM).

Histological analysis. Tumor sample fixation, and paraffin embedding, sectioning, and immunofluorescence staining were performed based on our previous publications $(14,15)$. Hematoxylin $\&$ eosin staining was conducted following a standard protocol.

Statistical analysis. All statistical analyses were performed as previously reported (14) The Shapiro-Wilk test was used to assess normal distribution. To verify the homogeneity of variances across between groups, the Bartlett's test was performed. For the parametric test for inter-group comparison, one-way ANOVA with Tukey's HSD for post-hoc analysis was conducted. The paired $t$-test using a parametric test was performed to compare the means between two related groups. All $p$-values were two-sided and a $p$-value of $<0.05$ was regarded as statistically significant.

\section{Results}

Effect of SFN, EVL, DOX on the osteosarcoma-PDOX. No statistical difference was observed in osteosarcoma PDOX growth between DOX and the control $(p=0.2)$. Treatment individually with SFN or EVL resulted in significant inhibition of osteosarcoma PDOX growth, but moderately, compared with the control $(p<0.001$ and $p<0.001$, respectively) or with DOX alone ( $p=0.005$ and $p=0.005$, respectively). However, the combination of SFN-EVL significantly regressed the osteosarcoma PDOX tumor (compared with control: $p<0.001$ or DOX alone: $p<0.001$ ). (Figures 2, 3A, B).

Effect of SFN, EVL, DOX on body weight. Mouse body weight was measured before treatment and after treatment. The body weight at the end of treatment of control mice, or mice treated with DOX alone or SFN alone, increased significantly compared with the initial body weight ( $p=0.004, p=0.01$, and $p=0.009$, respectively). We did find a significant difference in body weight among the other groups (Figure 3C). In addition, no side effects or animal deaths were observed in any treatment group.

Histology of the osteosarcoma in PDOX. The control tumor or tumor treated with DOX showed viable highly-dense cancer cells with a pleomorphic spindle-like phenotype. Osteosarcoma PDOX tumors treated with SFN alone or EVL contained viable cancer cells even though the cancer-cell density was lower compared to the control. Further, when osteosarcoma PDOX tumors were treated with the SFN-EVL combination, we observed the lowest cancer-cell density with necrotic cancer cells and degenerative scars in the stroma (Figure 4).

\section{Discussion}

In this study, we showed that a combination of SFN-EVL regressed a DOX-resistant osteosarcoma in a PDOX model. To the best of our knowledge, this is the first study that shows that an SFN-EVL combination is active in osteosarcoma.

Targeting major signaling pathways through combination therapies could improve the antitumor efficacy and minimize drug resistance after up-regulation of a secondary pathway as a result of treatment with single-agents. It has been reported that SFN together with PI-103 inhibits EGF-stimulated Ras/Raf/MAPK and PI3K/AKT/mTOR pathways (16). Even though SFN has upstream inhibitory effects on the MAPK/ERK pathway, it can also stimulate downstream components by upregulating mTORC2 activity, resulting in disease progression or induce resistance of the tumor to SFN (11). EVL, when combined with SFN, can minimize the stimulatory effect of SFN on mTORC2 by disassembling this protein complex (11). Furthermore, EVL has been shown to reduce the resistance to $\mathrm{SFN}$ in osteosarcoma because the AKT-mTOR pathway, which is the main target of EVL, has a role in resistance to SFN in osteosarcoma. This suggests the high synergy SFN-EVL combination on osteosarcoma (9). In the present study, we determined moderate efficacy of SFN or EVL alone and high efficacy of the SFN-EVL combination on a DOX-resistant osteosarcoma in a PDOX model.

We have established PDOX mouse model for all major cancers. We have shown that the PDOX model is more patient-like compared to subcutaneous patient-derived xenograft (PDX) models $(17,18)$. We have previously 
A

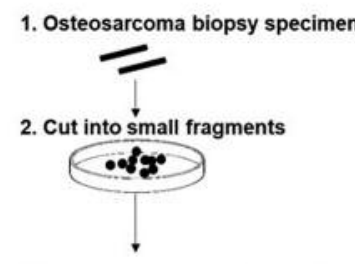

3. Tumor grown subcutaneously

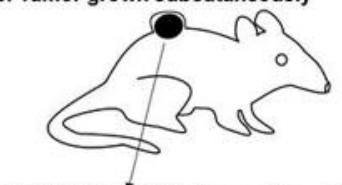

4. Divide tumor into fragments

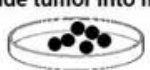

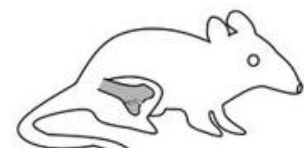

5. Osteotomy of the lateral condyle of the right distal femur

6. Orthotopic implantation of tumor fragments into distal femur

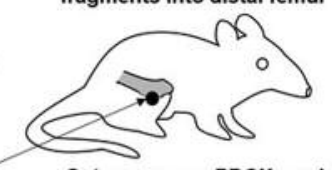

Osteosarcoma-PDOX model
B

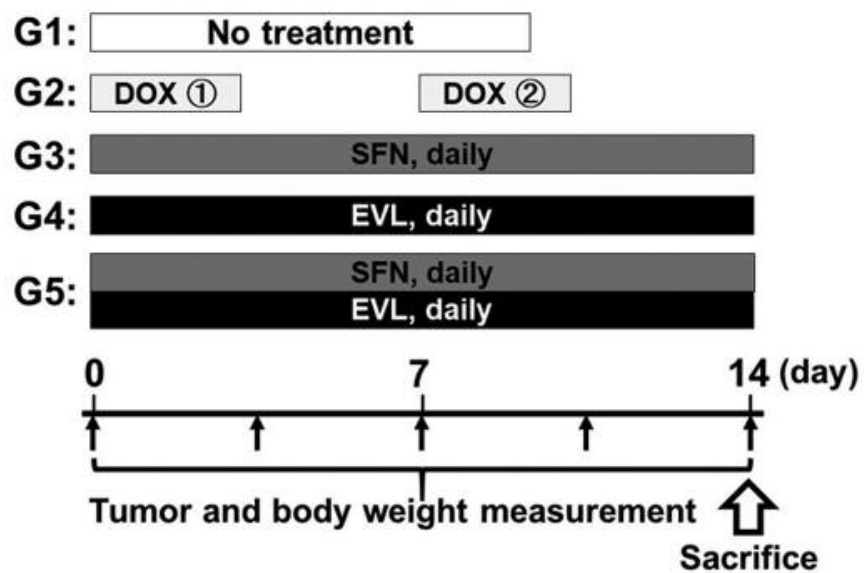

Figure 1. Establishment of the osteosarcoma PDOX model and treatment scheme. Schematic diagram of establishing the osteosarcoma PDOX model [modified after (15)] (A). Treatment schema (B).

A

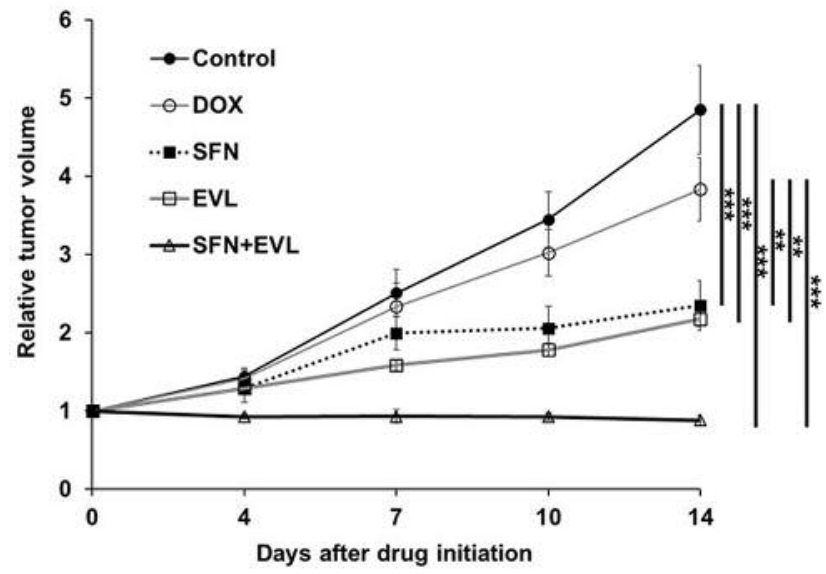

B

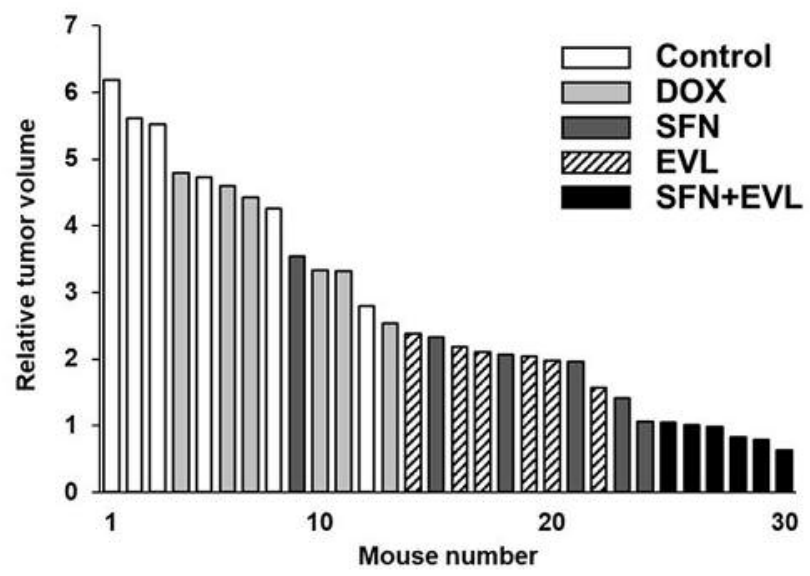

Figure 2. Efficacy of drugs on the tumor volume in the osteosarcoma PDOX model (A). Tumor volume at each time point is represented by line graphs after the initiation of treatment. Data are as tumor volume presented relative to the initial tumor volume for each treatment and the control group. Relative tumor volume at day 14 relative to the initial tumor volume for each mouse is presented in a waterfall plot (B). N=6 mice/group. ${ }^{* *} p<0.01 ; * * * p<0.001$. Error bars: \pm SEM.

demonstrated that the PDOX model retains the histopathological/molecular characteristics of the original tumor after transplantation in mice $(19,20)$. PDOX models provide a unique opportunity to derive precise and personalized treatment choices for sarcoma patients.

In conclusion, the present study uniquely demonstrates the power of the PDOX model in identifying effective treatment of osteosarcoma with an SFN and EVL combination. The fact that the SFN-EVL combination could regress the osteosarcoma PDOX tumor suggests clinical promise.

\section{Conflicts of Interest}

AntiCancer Inc. uses PDOX models for contract research. TH, NS, MK, $\mathrm{HO}, \mathrm{NY}, \mathrm{KH}, \mathrm{HK}, \mathrm{SM}, \mathrm{KI}$ and RMH are or were unsalaried associates of AntiCancer Inc. There are no other competing financial interests.

\section{Authors' Contributions}

Conception and design: TH and RMH. Acquisition of data: TH, NS, $\mathrm{KM}, \mathrm{HO}, \mathrm{NY}, \mathrm{KH} \mathrm{HK}, \mathrm{SM}$, and KI. Analysis and interpretation of data: TH, NS, KM, HO, NY, KH, HK, SM, KI, SPC, MB, SRS, HT, 


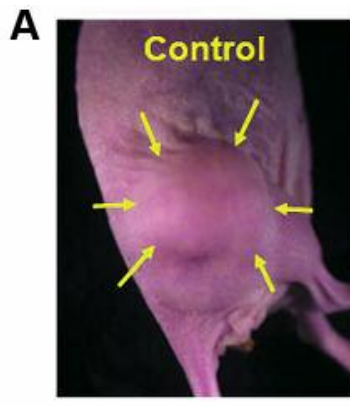

B

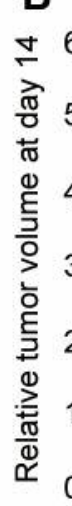

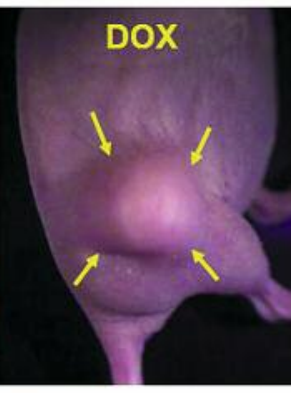

$\star \star \star$

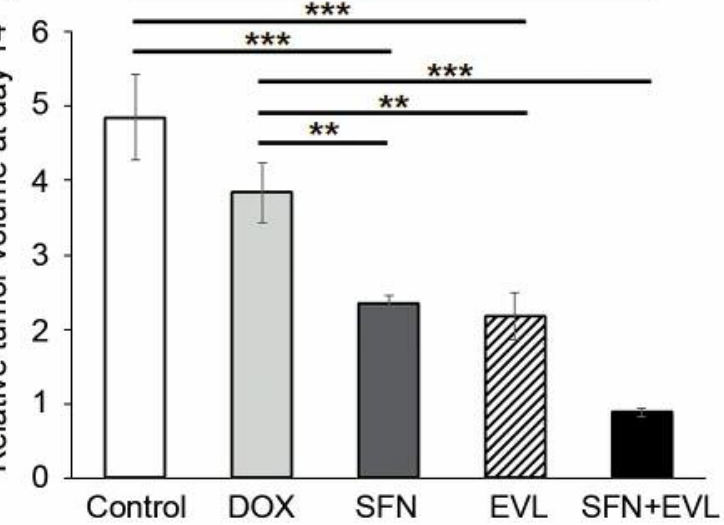

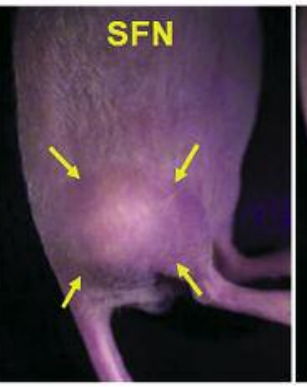
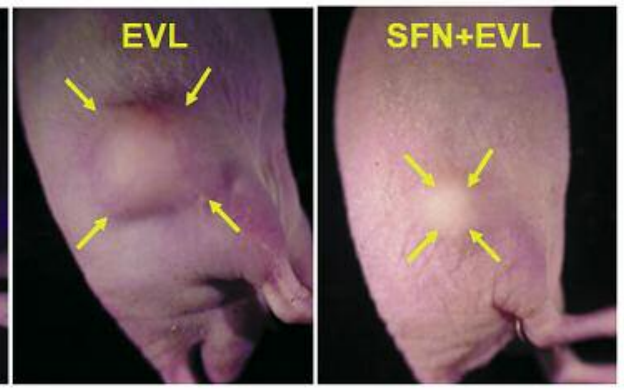

C

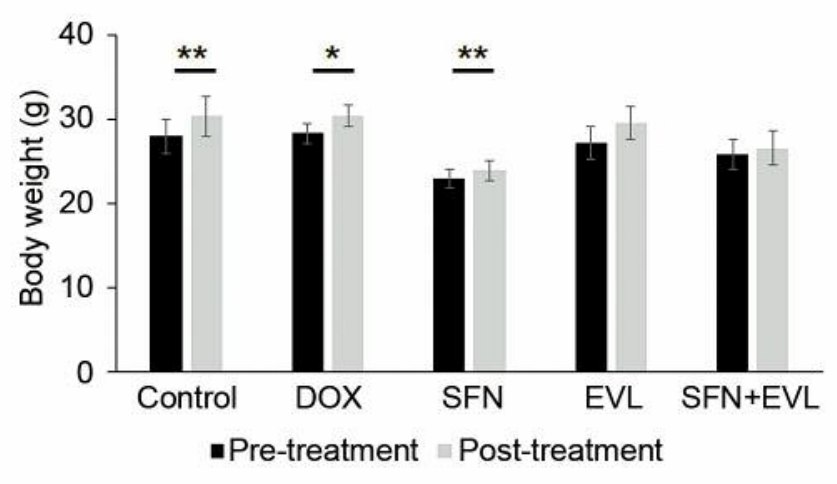

Figure 3. Representative photographs of the osteosarcoma PDOX mouse models from each treatment group at the end of treatment (A). The approximate margin of the tumors are indicated by yellow arrows. Bar graphs show relative tumor volume of each treatment group on day 14 (B). $N=6$ mice/group. ${ }^{*} p<0.01 ; * * * p<0.001$. Error bars: $\pm S E M$. (C) Effect of DOX, SFN, EVL, and SFN+EVL on mouse body weight at pre- and posttreatment times. $N=6$ mice/group. $* p<0.05 ; * *<<0.01$. Error bars: \pm SEM.
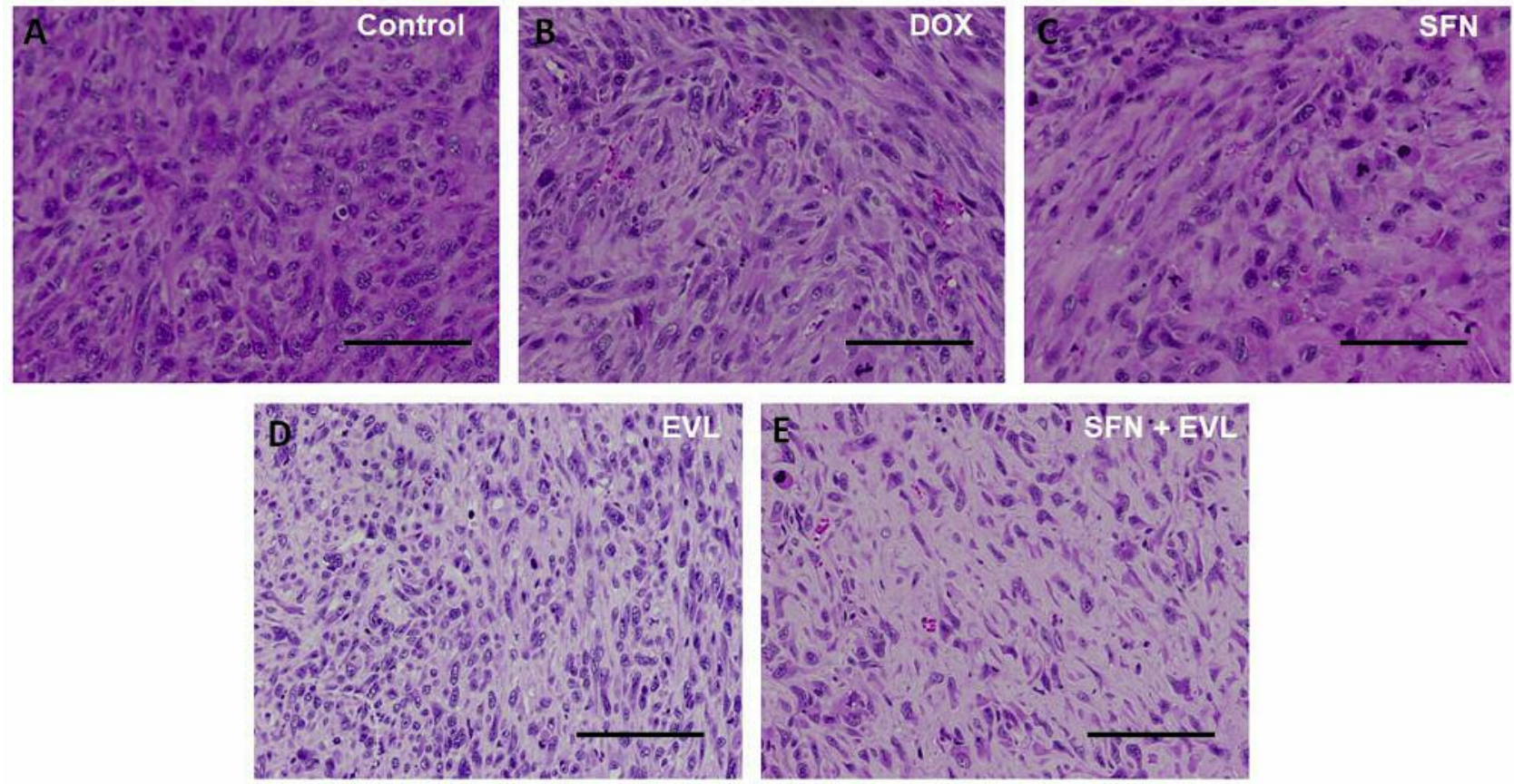

Figure 4. Tumor histology. Untreated control (A), DOX (B), SFN (C), EVL (D), combination treatment with SFN + EVL (E). Scale bars: $100 \mu$ m. 
and RMH. Writing, review, and/or revision of the manuscript: TH, RMH, HT, PB, and SRS.

\section{Acknowledgements}

This paper is dedicated to the memory of Reese Imhoff.

\section{References}

1 Misaghi A, Goldin A, Awad M and Kulidjian AA: Osteosarcoma: a comprehensive review. SICOT J 4: 12, 2018. PMID: 29629690. DOI: $10.1051 / \mathrm{sicotj} / 2017028$

2 Grignani G, Palmerini E, Dileo P, Asaftei SD, D’Ambrosio L, Pignochino Y, Mercuri M, Picci P, Fagioli F, Casali PG, Ferrari $\mathrm{S}$ and Aglietta M: A phase II trial of sorafenib in relapsed and unresectable high-grade osteosarcoma after failure of standard multimodal therapy: an Italian Sarcoma Group study. Ann Oncol 23(2): 508-516, 2012. PMID: 21527590. DOI: 10.1093/annonc/ mdr 151

3 Coventon J: A review of the mechanism of action and clinical applications of sorafenib in advanced osteosarcoma. J Bone Oncol 8: 4-7, 2017. PMID: 28828294. DOI: 10.1016/j.jbo.2017. 07.001

4 Yang J, Samsel PA, Narov K, Jones A, Gallacher D, Gallacher J, Sampson JR and Shen MH: Combination of everolimus with sorafenib for solid renal tumors in Tsc2(+/-) mice is superior to everolimus alone. Neoplasia 19(2): 112-120, 2017. PMID: 28092822. DOI: 10.1016/j.neo.2016.12.008

5 Hainsworth JD, Waterhouse DM, Penley WC, Shipley DL, Thompson DS, Webb CD and Anthony Greco F: Sorafenib and everolimus in advanced clear cell renal carcinoma: a phase I/II trial of the SCRI Oncology Research Consortium. Cancer Invest 31(5): 323-329, 2013. PMID: 23614653. DOI: 10.3109/ 07357907.2013.789900

6 Koeberle D, Dufour JF, Demeter G, Li Q, Ribi K, Samaras P, Saletti P, Roth AD, Horber D, Buehlmann M, Wagner AD, Montemurro M, Lakatos G, Feilchenfeldt J, Peck-Radosavljevic M, Rauch D, Tschanz B and Bodoky G; Swiss Group for Clinical Cancer Research (SAKK): Sorafenib with or without everolimus in patients with advanced hepatocellular carcinoma (HCC): a randomized multicenter, multinational phase II trial (SAKK 77/08 and SASL 29). Ann Oncol 27(5): 856-861, 2016. PMID: 26884590. DOI: 10.1093/annonc/mdw054

7 Mariniello B, Rosato A, Zuccolotto G, Rubin B, Cicala MV, Finco I, Iacobone M, Frigo AC, Fassina A, Pezzani R and Mantero F: Combination of sorafenib and everolimus impacts therapeutically on adrenocortical tumor models. Endocr Relat Cancer 19(4): 527-39, 2012. PMID: 22673336. DOI: 10.1530/ ERC-11-0337

8 Yoo C, Lee J, Rha SY, Park KH, Kim TM, Kim YJ, Lee HJ, Lee $\mathrm{KH}$ and Ahn JH: Multicenter phase II study of everolimus in patients with metastatic or recurrent bone and soft-tissue sarcomas after failure of anthracycline and ifosfamide. Invest New Drugs 31(6): 1602-1608, 2013. PMID: 24037083. DOI: 10.1007/s10637-013-0028-7

9 Grignani G, Palmerini E, Ferraresi V, D'Ambrosio L, Bertulli R, Asaftei SD, Tamburini A, Pignochino Y, Sangiolo D, Marchesi E, Capozzi F, Biagini R, Gambarotti M, Fagioli F, Casali PG, Picci P, Ferrari S and Aglietta M; Italian Sarcoma Group:
Sorafenib and everolimus for patients with unresectable highgrade osteosarcoma progressing after standard treatment: a nonrandomised phase 2 clinical trial. Lancet Oncol 16(1): 98-107, 2015. PMID: 25498219. DOI: 10.1016/S1470-2045(14)71136-2

10 Pawaskar DK, Straubinger RM, Fetterly GJ, Hylander BH, Repasky EA, Ma WW, Jusko WJ: Synergistic interactions between sorafenib and everolimus in pancreatic cancer xenografts in mice. Cancer Chemother Pharmacol 71(5): 1231-1240, 2013. PMID: 23455452. DOI: 10.1007/s00280-013-2117-x

11 Pignochino Y, Dell'Aglio C, Basiricò M, Capozzi F, Soster M, Marchiò S, Bruno S, Gammaitoni L, Sangiolo D, Torchiaro E, D'Ambrosio L, Fagioli F, Ferrari S, Alberghini M, Picci P, Aglietta $\mathrm{M}$ and Grignani G: The combination of sorafenib and everolimus abrogates mTORC1 and mTORC2 upregulation in osteosarcoma preclinical models. Clin Cancer Res 19(8): 211721131, 2013. PMID: 23434734. DOI: 10.1158/1078-0432.CCR$12-2293$

12 Miyake K, Kiyuna T, Kawaguchi K, Higuchi T, Oshiro H, Zhang Z, Wangsiricharoen S, Razmjooei S, Li Y, Nelson SD, Murakami T, Hiroshima Y, Matsuyama R, Bouvet M, Chawla SP, Singh SR, Endo I and Hoffman RM: Regorafenib regressed a doxorubicinresistant Ewing's sarcoma in a patient-derived orthotopic xenograft (PDOX) nude mouse model. Cancer Chemother Pharmacol 83(5): 809-815, 2019. PMID: 30758647. DOI: 10.1007/s00280-019-03782-w

13 Higuchi T, Miyake K, Oshiro H, Sugisawa N, Yamamoto N, Hayashi K, Kimura H, Miwa S, Igarashi K, Chawla SP, Bouvet M, Singh SR, Tsuchiya H and Hoffman RM: Trabectedin and irinotecan combination regresses a cisplatinum-resistant osteosarcoma in a patient-derived orthotopic xenograft nudemouse model. Biochem Biophys Res Commun 513(2): 326-333, 2019. PMID: 30955860. DOI: 10.1016/j.bbrc.2019.03.191

14 Higuchi T, Sugisawa N, Miyake K, Oshiro H, Yamamoto N, Hayashi K, Kimura H, Miwa S, Igarashi K, Bouvet M, Singh SR, Tsuchiya $\mathrm{H}$ and Hoffman RM: The combination of olaratumab with doxorubicin and cisplatinum regresses a chemotherapy-resistant osteosarcoma in a patient-derived orthotopic xenograft mouse model. Transl Oncol 12(9): 12571263, 2019. PMID: 31299622. DOI: 10.1016/j.tranon.2019. 06.002

15 Higuchi T, Miyake K, Sugisawa N, Oshiro H, Zhang Z, Razmjooei S, Yamamoto N, Hayashi K, Kimura H, Miwa S, Igarashi K, Bouvet M, Singh SR, Tsuchiya H and Hoffman RM: Olaratumab combined with doxorubicin and ifosfamide overcomes individual doxorubicin and olaratumab resistance of an undifferentiated soft-tissue sarcoma in a PDOX mouse model. Cancer Lett 451: 122-137, 2019. PMID: 30867142. DOI: 10.1016/j.canlet.2019.03.003

16 Gedaly R, Angulo P, Hundley J, Daily MF, Chen C, Koch A and Evers BM: PI-103 and sorafenib inhibit hepatocellular carcinoma cell proliferation by blocking Ras/Raf/MAPK and $\mathrm{PI} 3 \mathrm{~K} / \mathrm{AKT} / \mathrm{mTOR}$ pathways. Anticancer Res 30(12): 4951-4958, 2010. PMID: 21187475.

17 Igarashi K, Kawaguchi K, Kiyuna T, Murakami T, Miwa S, Nelson SD, Dry SM, Li Y, Singh A, Kimura H, Hayashi K, Yamamoto N, Tsuchiya H, Eilber FC and Hoffman RM: Patientderived orthotopic xenograft (PDOX) mouse model of adult rhabdomyosarcoma invades and recurs after resection in contrast to the subcutaneous ectopic model. Cell Cycle 16(1): 91-94, 2017. PMID: 27830986. DOI: 10.1080/15384101.2016.1252885 
18 Hiroshima Y, Maawy A, Zhang Y, Zhang N, Murakami T, Chishima T, Tanaka K, Ichikawa Y, Bouvet M, Endo I and Hoffman RM: Patient-derived mouse models of cancer need to be orthotopic in order to evaluate targeted anti-metastatic therapy. Oncotarget 7(44): 71696-71702, 2016. PMID: 277659 34. DOI: 10.18632/oncotarget.12322

19 Yamamoto M, Zhao M, Hiroshima Y, Zhang Y, Shurell E, Eilber FC, Bouvet M, Noda M and Hoffman RM: Efficacy of tumortargeting salmonella A1-R on a melanoma patient-derived orthotopic xenograft (PDOX) nude-mouse model. PloS One 11(8): e0160882, 2016. PMID: 27500926. DOI: 10.1371/journal. pone. 0160882

20 Igarashi K, Kawaguchi K, Li S, Han Q, Tan Y, Murakami T, Kiyuna T, Miyake K, Miyake M, Singh AS, Eckardt MA, Nelson SD, Russell TA, Dry SM, Li Y, Yamamoto N, Hayashi K,
Kimura H, Miwa S, Tsuchiya H, Singh SR, Eilber FC and Hoffman RM: Recombinant methioninase in combination with doxorubicin (DOX) overcomes first-line DOX resistance in a patient-derived orthotopic xenograft nude-mouse model of undifferentiated spindle-cell sarcoma. Cancer Lett 417: 168-173, 2018. PMID: 29306021. DOI: 10.1016/j.canlet.2017.12.028

Received August 6, 2019

Revised August 12, 2019

Accepted August 14, 2019 